\title{
The Detection of Glaucomatous Visual Field Defects by Oculo-Kinetic Perimetry: Which points are best for screening?
}

\author{
B. E. DAMATO, J. AHMED, D. AllAN, E. McClURE, J. L. JAY. \\ Glasgow
}

\begin{abstract}
Summary
Oculo-kinetic perimetry (OKP) uses the patient's ocular movements to position a test stimulus in the visual field. By virtue of its simplicity and low cost, this test could be useful in the screening of glaucoma. The general purpose OKP chart, however, which tests 100 points in the central $25^{\circ}$ field, is too time-consuming for this purpose, taking approximately 4-7 minutes per eye. Accordingly, this study was performed to identify the points most likely to detect patients with glaucoma. Fifty-one eyes with glaucomatous visual field defects and 51 non-glaucomatous eyes of age matched individuals were tested by conventional and oculo-kinetic perimetry. At least one of only six points were missed by $82.4 \%$ of glaucomatous eyes and by $9.8 \%$ of nonglaucomatous eyes. These results, although falling short of the ideal efficiency, are comparable with data published by other research groups using computerised equipment and suggest that, with further development, a specialised OKP chart testing only a small number of points might be valuable in screening for glaucoma.
\end{abstract}

The role of visual field examination in glaucoma screening is limited by the lack of a test that is reliable yet quick, simple, and inexpensive. One test that could meet all these requirements is oculo-kinetic perimetry (OKP). ${ }^{1}$ The test is performed using a series of numbered fixation targets to place a single fixed test stimulus into known positions in the visual field. The general purpose OKP chart, which examines 100 points in the central $25^{\circ}$ field, gives essentially the same results as conventional perimeters. ${ }^{2}$ Although the time taken to test 100 points is comparable with that taken by other methods, it is too long for screening purposes.

This article describes a study in which we identified the points on the OKP chart that are most likely to detect glaucomatous visual field defects.

\section{Materials, Methods and Patients}

Age matched glaucomatous and nonglaucomatous eyes were randomly examined by oculo-kinetic and conventional perimetry on the same day. Details of the patient's age, sex, corrected visual acuity and cooperation were recorded. In addition, full ocular examination was performed when any unexpected visual field defects were discovered.

Glaucomatous eyes were included in this study if all of the following features were present: (i) unequivocal glaucomatous visual field loss; (ii) other evidence of glaucoma (ie, pathological optic disc cupping, elevated intra- 


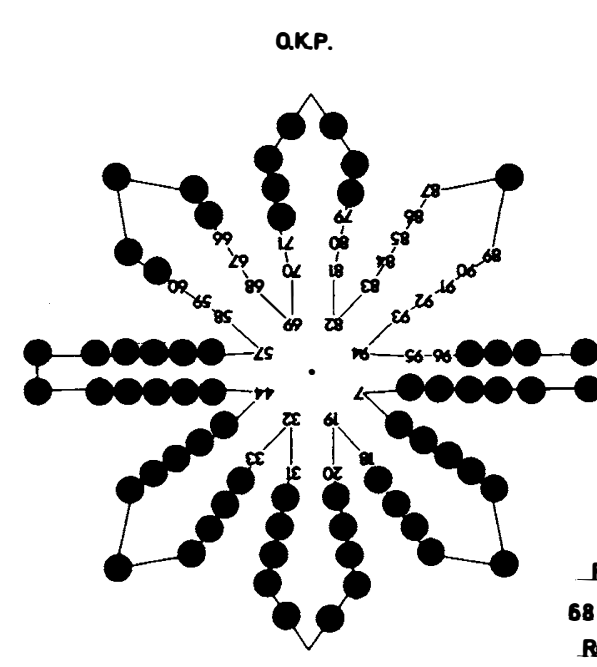

Drot anen at 3/1000

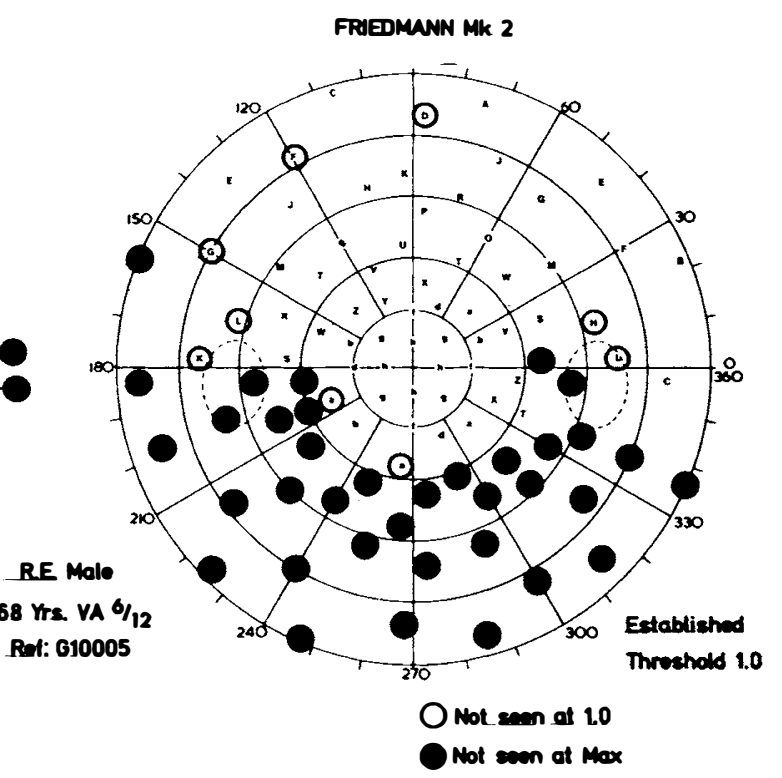

Fig. 1. Right visual field of a 68 year old man with glaucoma, plotted with (a) the 100-number OKP chart and (b) the Friedmann Mk 2 Visual Field Analyser.

ocular pressure); (iii) absence of other significant ocular disease; (iv) a visual acuity of $6 / 18$ or better.

The control group was drawn from (i) patients attending a nearby Dermatology Ward, (ii) hospital staff, (iii) relatives and friends of patients, and (iv) patients with unilateral non-glaucomatous disease affecting the fellow eye.

Conventional perimetry using (i) the Friedmann Visual Field Analyser Mark II, or (ii) the Dicon Autoperimeter 3000 or (iii) the Tubinger Oculus perimeter was performed by someone experienced in perimetry according to the manufacturer's instructions.

Oculo-kinetic perimetry was performed in a brightly lit room, with a screen illuminance of 200 lux. The 100-point OKP test chart has been described previously. ${ }^{1,2}$ It consisted of a white tangent screen, with 100 peripheral numbered fixation points and a central black test stimulus, which was 2,3 or $5 \mathrm{~mm}$ in diameter and attached to the screen by a pin. Seated one metre from the chart, with one eye occluded, the patient looked at each number in turn and, as the operator covered and uncovered the spot with a white card, indicated whenever this stimulus appeared. The numbers associated with non-awareness of the test stimulus were marked off on a miniature version of the OKP chart on a record sheet, which was inverted at the end of the test so that the plotted results were comparable with those obtained conventionally (Figure 1). The principles of the examination were demonstrated to the patient and the level of cooperation simultaneously assessed by first testing the blindspot. Any missed points were re-tested, first using the same stimulus, and then with progressively larger

Table I. Ages of patients with glaucoma and non-glaucomatous controls.

\begin{tabular}{lcccccccc}
\hline $\begin{array}{l}\text { Age group } \\
\text { (years) }\end{array}$ & $20-29$ & $30-39$ & $40-49$ & $50-59$ & $60-69$ & $70-79$ & $80-89$ & Total \\
\hline Glaucoma & 2 & 7 & 8 & 10 & 11 & 12 & 1 & 51 \\
Control & 1 & 7 & 8 & 10 & 11 & 12 & 2 & 51 \\
\hline
\end{tabular}


Table II. Severity of glaucomatous visual field defects, categorised using Aulhorn and Karmeyer's Classification, in 51 eyes tested with the 100-number OKP chart.

\begin{tabular}{llc}
\hline Grade & Definition & Number \\
\hline I & $\begin{array}{l}\text { Relative defects only } \\
\text { II }\end{array}$ & 32 \\
III & $\begin{array}{l}\text { defects separate from blindspot } \\
\text { Arcuate absolute defects }\end{array}$ & 14 \\
IV & $\begin{array}{l}\text { Extensive to blingspot } \\
\text { altitudinal defects }\end{array}$ & 4 \\
V & Visual field loss involving fixation & 0 \\
\hline
\end{tabular}

stimuli so that the depth of the visual field defects could be evaluated.

Once the most appropriate spot size had been determined, all eyes were examined with this stimulus. The OKP points most specific for glaucoma were selected by first eliminating all OKP points'missed by more than $5 \%$ of the control eyes, and then identifying the points which detected most eyes with glaucomatous visual field defects.

\section{Results}

The mean ages of the patients with glaucomatous and non-glaucomatous eyes were 57.3 years and 54.4 years respectively (Table I). The severity of the glaucomatous visual field loss was graded according to Aulhorn and Karmeyer's Classification ${ }^{3}$ as shown in Table II. Most patients had early visual field loss.

The isopters at which the $2 \mathrm{~mm}, 3 \mathrm{~mm}$ and $5 \mathrm{~mm}$ black stimuli were missed by more than five per cent of 51 normal eyes are shown in Figure 2. The $3 \mathrm{~mm}$ stimulus was found to be approximately equivalent to the $100 \mathrm{Asb}$ stimulus of the Dicon perimeter and the $1.0 \mathrm{w} 0.5$ stimulus of the Tubingen Oculus perimeter. It was selected for further study because its five per cent isopter was located immediately peripheral to the areas in the visual field where arcuate glaucomatous defects are known to occur.

In the 51 healthy eyes examined with the 100 -number OKP test chart and the 3/1000 black test stimulus, 46 points were missed by five per cent of eyes or fewer. These were situated at or within the central $15^{\circ}$ field. The most sensitive points for glaucoma were located at $12.5^{\circ}$ of eccentricity in the superior field and at $15^{\circ}$ infero-nasally and nasally (Fig. 3).

The cumulative percentages of glaucomatous and non-glaucomatous eyes missing one or more points are shown in Figure 4. The best point corresponded to number 80 on the OKP chart, which was missed by $45 \%$ of all eyes with glaucomatous visual field defects and $3.9 \%$ of controls. At least one of the two points, 80 and 96 , were missed by $66 \%$ of the glaucomatous eyes and by $7.8 \%$ of the controls. Six points detected $82.4 \%$ of eyes with glaucomatous visual field loss with a false positive rate of $9.8 \%$. When more than six points were tested, there was a relatively small gain in the detection rate compared to the large increase in the false positives, as indicated by the more gentle slope in the curve.

\section{Discussion}

There is a need for a simple, yet efficient, visual field test that could be performed rapidly and conveniently when screening for glaucoma. This study suggests that an OKP screening chart testing only six points could detect over $80 \%$ of eyes with glaucomatous visual field loss.

Comparison of the results obtained from glaucomatous eyes with those of age matched normal eyes showed that the points most likely to detect glaucomatous visual field loss

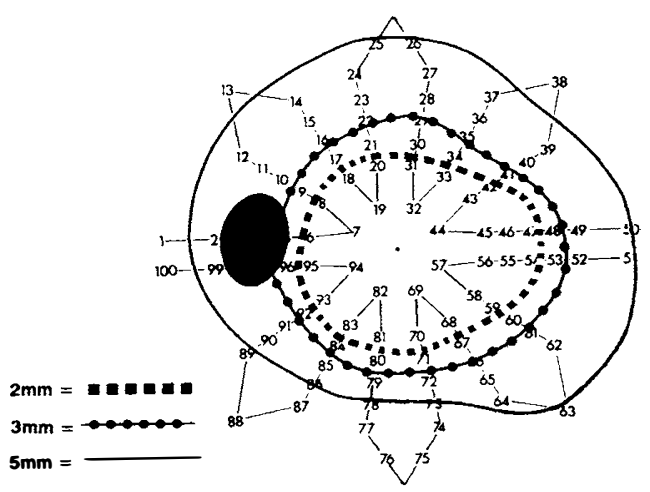

Fig. 2. Isopters on the one meter 100 number $O K P$ chart at which $2 \mathrm{~mm}, 3 \mathrm{~mm}$ and $5 \mathrm{~mm}$ spherical black stimuli were missed by more than five per cent of 51 normal right eyes (NB: Field is inverted). 


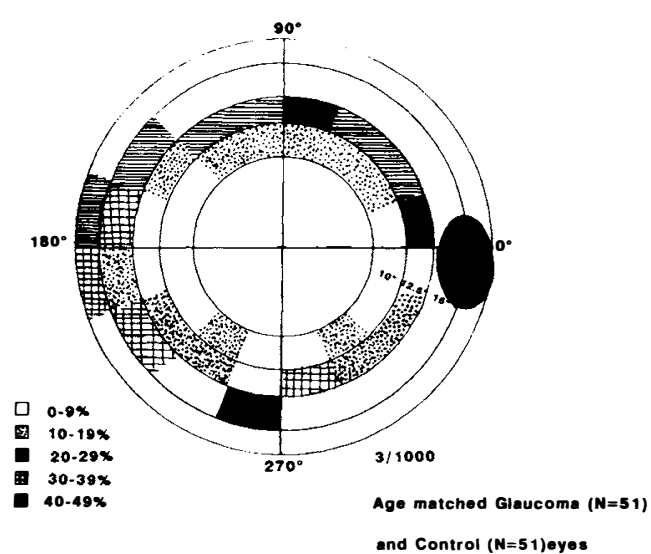

Fig. 3. Distribution of glaucomatous visual field defects measured by Oculo-Kinetic Perimetry. The most specific points were situated at 12.5 degrees in the superior field and at 15 degrees inferonasally and nasally.

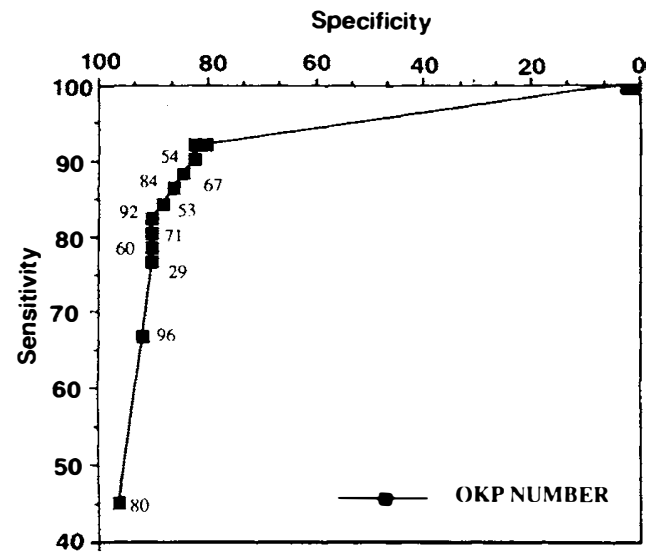

Fig. 4. Cumulative percentages of glaucomatous and non-glaucomatous eyes missing one or more points tested with the 3/1000 black stimulus on the 100-number $O K P$ chart. The relative operating curve (ROC) predicts that at least one of six points would be missed by $84.4 \%$ of eyes with glaucoma and by $9.8 \%$ of nonglaucomatous eyes.

are located in the superior paracentral visual field and in the infero-nasal field. It is likely that the points situated $15^{\circ}$ superiorly were non-specific because of lid artefact and that the infero-temporal points were excluded because they coincided with angioscotomata. The distribution of glaucomatous defects as measured by OKP, and using a black stimulus, was remarkably similar to that described previously by other research groups using a variety of conventional perimeters. ${ }^{3-10}$
Although not identified as important by our analysis, the testing of additional points close to fixation may be necessary because it is known that glaucomatous visual field loss could commence centrally, ${ }^{11}$ particularly in low-tension glaucoma. ${ }^{12}$ Although some glaucomatous eyes have isolated peripheral defects, ${ }^{13-14}$ the benefits of detecting such cases would be outweighed by the disproportionately high false positive rate that would undoubtedly occur.

The false positive rate of almost $10 \%$ predicted in this study is greater than reported in other studies. This is probably because we selected control eyes without prior knowledge of the ocular status or the subjects' level of cooperation, unlike other workers who prescreened their control subjects; ${ }^{15,16}$ we may have achieved similar results if we had used the same selection criteria for our controls as these workers. In any event, visual field loss caused by glaucoma is rare in the community compared to that caused by other conditions, such as amblyopia, uncorrected ametropia, cataract and macular disease. ${ }^{17}$ When screening for glaucoma by perimetry, therefore, additional ophthalmic examination by tonometry and ophthalmoscopy is required to prevent the referral of overwhelming numbers of false positives to the ophthalmologist. In this respect, the OKP chart is comparable to much more complex methods of visual field analysis.

In conclusion, this study has identified six points in the visual field which, if tested by oculo-kinetic perimetry using a 3/1000 black stimulus or equivalent, should detect a high proportion of eyes with glaucoma. There is scope for further research aimed at the production of an OKP test chart specifically for this purpose. Such a test could facilitate the recognition of glaucoma, thereby stimulating greater interest in screening for this disease.

We gratefully acknowledge the financial assistance of the Visual Research Trust and the artwork of Mrs A Currie, Mrs D. Aitken, and Mrs S. Cameron.

\section{References}

${ }^{1}$ Damato BE: Oculo-Kinetic Perimetry: a simple visual field test for use in the community $\mathrm{BrJ}$ Ophthalmol 1985, 69: 927-31.

${ }^{2}$ Alvarez E, Damato BE, Jay JL, McClure E: Com- 
parative evaluation of oculokinetic perimetry and conventional perimetry in glaucoma, $\mathrm{Br} J$ Ophthalmol 1988, 72: 258-62.

${ }^{3}$ Aulhorn E and Karmeyer H: Frequency distribution in early glaucomatous visual field defects. Doc Ophthalmol Proc Ser 1977, 14: 75-83.

${ }^{4}$ Furuno $\mathrm{F}$ and Matsuo $\mathrm{H}$ : Early stage progression in glaucomatous visual field changes. Doc Ophthalmol Proc Ser 1979, 19: 247-53.

${ }^{5}$ Kosaki H: The earliest visual field defect (IIa stage) in glaucoma by kinetic perimetry. Doc Ophthalmol Proc Ser 1979, 19: 255-9.

${ }^{6}$ Coughlan $\mathrm{M}$ and Friedmann AI: The frequency distribution of early visual field defects in glaucoma. Doc Ophthalmol Proc Ser 1981, 26: 345-9.

${ }^{7}$ Hart WM and Becker B: The onset and evolution of glaucomatous visual field defects. Ophthalmology 1982, 89: 268-79.

${ }^{8}$ Heijl $\mathrm{A}$ and Lundqvist L: The frequency distribution of earliest glaucomatous visual field defects documented by automatic perimetry. Acta Ophthalmol 1984, 62: 658-64.

${ }^{9}$ Rabin S, Kolesar P, Podos SM, Wilensky JT: A visual field screening protocol for glaucoma. Am J Ophthalmol 1981, 92: 530-5.
${ }^{10}$ Henson DB, Chauhan BC, Hobley A: Screening for glaucomatous visual field defects: the relationship between sensitivity, specificity and the number of test locations. Ophthal Physiol Opt 1988, 8: 123-7.

${ }^{11}$ Pickett JE, Terry SA, O'Connor PS, O'Hara M: Early loss of central visual acuity in glaucoma. Ophthalmology 1985, 92: 891-6.

12 Caprioli J and Spaeth GL: Comparison of visual field defects in the low-tension glaucomas with those in the high-tension glaucomas. Am J Ophthalmol 1984, 97: 730-7.

${ }^{13}$ Caprioli J and Spaeth GL: Static threshold examination of the peripheral nasal field in glaucoma. Arch Ophthalmol 1985, 103: 1150-4.

${ }^{14}$ Stewart WC, Shields B, Ollie A R: Peripheral visual field testing by automated kinetic perimetry in glaucoma. Arch Ophthalmol 1988, 106: 202-6.

${ }^{15}$ Rock W J, Drance SM, Morgan RW: Visual field screening in glaucoma. Arch Ophthalmol 1973, 89: $287-90$.

${ }^{16}$ Enger C and Sommer A: Recognizing glaucomatous field loss with the Humphrey STATPAC. Arch Ophthalmol 1987, 105: 1355-7.

17 Graham PA: Screening for chronic glaucoma. Proc $R$ Soc Med 1966, 59: 1215-20. 